Proceedings of the Edinburgh Mathematical Society (2006) 49, 71-86 (C)

DOI:10.1017/S0013091504001075 Printed in the United Kingdom

\title{
DIFFRACTIVE GEODESICS OF A POLYGONAL BILLIARD
}

\author{
LUC HILLAIRET \\ UMPA ENS-Lyon, 46 allée d'Italie, 69364 Lyon Cedex 7, \\ France (lhillair@umpa.ens-lyon.fr)
}

(Received 21 September 2004)

\begin{abstract}
We define the notion of diffractive geodesic for a polygonal billiard or, more generally, for a Euclidean surface with conical singularities. We study the local geometry of the set of such geodesics of given length and we relate it to a number that we call classical complexity. This classical complexity is then computed for any diffractive geodesic. As an application we describe the set of periodic diffractive geodesics as well as the symplectic aspects of the 'diffracted flow'.
\end{abstract}

Keywords: polygonal billiard; diffractive orbits; conical singularities

2000 Mathematics subject classification: Primary 37D50

\section{Introduction}

The propagation of singularities on a smooth Riemannian manifold states that the singularities of one solution of the wave equation propagate along the geodesics. This theorem has been proved in more general settings such as manifolds with a smooth boundary, with conical singularities, polygonal domains (see $[\mathbf{5}, \mathbf{6}, \mathbf{1 1}]$ ). In all these cases the propagation of singularities is true, provided that one takes the suitable generalization of geodesics, i.e. broken (or reflective) geodesics in the boundary case, diffractive geodesics in the case of conical singularities. In order to understand more precisely the propagator of the wave equation, it is then very helpful to know how these 'generalized' geodesics behave. One important issue is the description of the so-called geometric wavefront, which consists in the endpoints of all the possible geodesics emanating from a given starting point. The aim of this paper is to answer this question for the generalized (here diffractive) geodesics of a Euclidean surface with conical singularities (a setting which includes polygonal billiards). As stated previously, this study is principally motivated by the description of the wave propagator on such surfaces. However, since the notion of a diffractive geodesic is closely related to that of a generalized diagonal (introduced by Katok in [8]), we also believe that this paper can provide some more understanding of the dynamical properties of polygonal billiards. To this purpose, we also remark here that the results we obtain are independent of whether or not the polygon is rational.

In the rest of the paper, $M$ will always be either a Euclidean surface with conical singularities $[\mathbf{1 0}]$ or a polygonal domain in $\mathbb{R}^{2}$. We will begin by defining the set of 
(possibly) diffractive geodesics of $M$ and we will call $\Gamma_{T}(M)$ the set of all the geodesics of length $T$. Seeing an element of $\Gamma_{T}(M)$ as a mapping from $[0, T]$ in $M$, this set comes naturally equipped with a topology. Given $[p]$, any ordered sequence of conical points, we will also define $\Gamma_{T}^{[p]}(M)$ as the geodesics of length $T$ that go through the conical points prescribed by $[p]$. The local geometry of $\Gamma_{T}(M)$ near a geodesic $g$ is described by the number of strata $\Gamma_{T}^{[p]}$ to which $g$ is adherent. This number, which we call classical complexity (see Definition 3.1) is the central object of this paper. The main result that we obtain is the following theorem (see Theorem 3.12), which computes the classical complexity of a geodesic $g$ from the sequence of its diffraction angles.

Theorem 1.1. Let $g$ be a geodesic of length $T$ with $n$ diffractive points and such that its sequence of diffraction angles is written as

$$
(\underbrace{\varepsilon_{0} \pi, \ldots, \varepsilon_{0} \pi}_{k_{0}}, \beta_{g, l_{0}}, \ldots, \beta_{g, l_{1}}, \underbrace{\varepsilon_{1} \pi, \ldots, \varepsilon_{1} \pi}_{k_{1}}), \quad \beta_{g, l_{i}} \neq \varepsilon_{i} \pi .
$$

Then one of the following holds:

(i) if $k_{0}+k_{1}<n$, then $c_{\mathrm{c}}(g)=\left(k_{0}+1\right)\left(k_{1}+1\right)$;

(ii) if $k_{0}+k_{1}=n$ and $k_{0} k_{1} \neq 0$, then $c_{\mathrm{c}}(g)=\left(k_{0}+1\right)\left(k_{1}+1\right)$;

(iii) if $k_{0}+k_{1}=n$ and $k_{0} k_{1}=0$, then $c_{\mathrm{c}}(g)=\frac{1}{2} n(n+1)+1$.

We also give two important applications when aiming at a trace formula for such surfaces: the description of the set of periodic orbits and the symplectic interpretation of the geometrical wavefront.

The paper is organized as follows. In the next section we will introduce the geometrical setting and the diffractive geodesics; we will also establish the topological nature of $\Gamma_{T}(M)$. In $\S 3$ we will define the notion of classical complexity. We will then give some useful examples and, finally, we will compute the classical complexity of any given diffractive geodesic. Section 4 will be devoted to applications.

\section{Diffractive geodesics}

\subsection{Geometrical setting}

The notion of a Euclidean surface with conical singularities (ESCS) is defined in [10]. We recall that $M$ is an ESCS if $M$ can be partitioned in two sets $M=M_{0} \cup P$, where $M_{0}$ is a (non-complete) Riemannian surface that is locally isometric to the Euclidean plane. The set $P$ is discrete and, in the neighbourhood of each $p_{i} \in P, M$ is locally modelled on the Euclidean cone of total angle $\alpha_{i}$. Throughout the paper, the ESCS will always be assumed to be complete.

\section{Examples.}

(i) The Euclidean cone of angle $\alpha$ (denoted by $\mathcal{C}_{\alpha}$ ) is an ESCS. We denote by $p$ the tip of the cone. The smooth part $\check{\mathcal{C}}_{\alpha}$ (where $\left.\check{\mathcal{C}}_{\alpha}=\mathcal{C}_{\alpha} \backslash\{p\}\right)$ is globally parametrized by $(r, x) \in(0, \infty) \times \mathbb{R} / \alpha \mathbb{Z}$ with the metric $\mathrm{d} r^{2}+r^{2} \mathrm{~d} x^{2}$. 

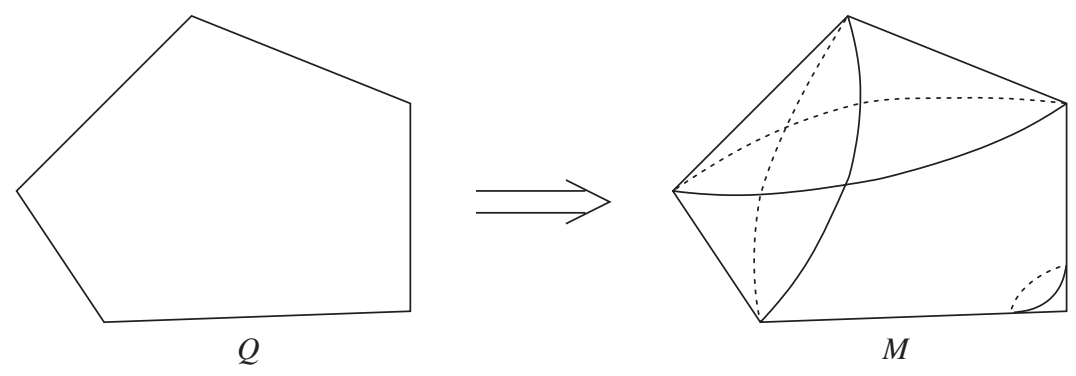

Figure 1. Doubling a polygon.

(ii) A simple way of constructing an ESCS is by glueing Euclidean polygons along sides of same length. For instance, taking two copies of a polygon $Q$ and glueing them along the sides gives an ESCS where each conical point corresponds to a vertex of the polygon. The angle $\alpha_{i}$ is then twice the corresponding angle of the polygon shown in Figure 1. This doubling method is standard in the study of billiards and was first used by Birkhoff.

(iii) A translation surface is a surface with conical singularities, and equipped with an atlas (outside the singularities) such that the transition functions are given by translations (see [7] for a more precise definition). Such a translation surface is automatically an ESCS, in particular the one that is associated with a rational polygon.

We now want to define the geodesics of such a surface. We denote by $P_{\mathrm{r}}$ the subset of $P$ consisting of the conical points $p_{i}$ such that $\alpha_{i}=2 \pi / k$ (and we let $P_{\mathrm{d}}$ be the complement of $P_{\mathrm{r}}$ in $P$, the conical points in $P_{\mathrm{d}}$ will be called diffractive). Locally, near any point of $M_{0} \cup P_{\mathrm{r}}, M$ is (up to a finite covering) isometric to $\mathbb{R}^{2}$. There is also no ambiguity in defining the geodesics as the projection of the geodesics of $\mathbb{R}^{2}$ (i.e. straight lines). This defines the non-diffractive geodesics of $M$. Any non-diffractive geodesic either can be extended to infinity or ends at a diffractive conical point in finite time. Since we need to extend such a geodesic, we give the following definition.

Definition 2.1. A geodesic of an ESCS will be a mapping $g:[0, \infty) \rightarrow M$ such that

(i) $g^{-1}\left(P_{\mathrm{d}}\right)$ is discrete,

(ii) if $g(t) \in M_{0} \cup P_{\mathrm{r}}$, there exists $\varepsilon$ such that the restriction of $g$ to $(t-\varepsilon, t+\varepsilon)$ parametrizes by arc length a non-diffractive geodesic.

\section{Remarks.}

(1) We have found it more convenient to define a geodesic as a mapping and not as a curve.

(2) On the Euclidean cone of angle $\alpha(\alpha \neq 2 \pi / k)$, this definition leads to two types of geodesics: 
(i) the non-diffractive ones, which are straight lines avoiding the tip of the cone,

(ii) the diffractive ones, which are formed by the juxtaposition of an incoming and of an outgoing ray, i.e. which are parametrized by

$$
g^{\gamma}\left(T_{1}\right)=p, \quad g^{\gamma}(t)= \begin{cases}\left(T_{1}-t, x_{\mathrm{i}}\right) & t<T_{1}, \\ \left(t-T_{1}, x_{\mathrm{o}}\right) & t>T_{1} .\end{cases}
$$

For such a geodesic, the angle $\beta=x_{\mathrm{o}}-x_{\mathrm{i}}$ is called the angle of diffraction; it belongs to $\mathbb{R} / \alpha \mathbb{Z}$. This angle of diffraction depends on the orientation of $\mathcal{C}_{\alpha}$.

(3) The reason for this definition is the theorem of propagation of singularities on a Euclidean cone $[\mathbf{1}, \mathbf{2}]$.

This definition implies that, near a conical point, $g$ parametrizes a geodesic of the corresponding cone; it allows us to define an angle of diffraction for each value of $t$ such that $g(t) \in P_{\mathrm{d}}$.

Remark 2.2. These angles $\beta$ depend on the orientation of $M$ near the conical points. When $M$ is not orientable, there is no preferable choice. In this case, we choose an orientation near the beginning of the geodesic, we transport it along and we consider the angles relative to this compatible orientation. Changing the orientation at the beginning will then multiply all the angles of diffraction by -1 . This has no consequences for us since the information we need is invariant under this change (see (3.2), below).

Notation. Along a geodesic $g$ we will denote by $p_{g, i}$ the $i$ th diffractive conical point, $t_{g, i}$ the time at which the diffraction occurs, and $\beta_{g, i}$ the $i$ th angle of diffraction. We will also denote by $[p]_{g}$ the sequence of diffractive points along $g$.

There is a globally defined distance $d$ on $M$ that is obtained by minimizing the length of curves. Locally, this distance coincides with that of the plane or of the corresponding Euclidean cone. We note that here a diffractive geodesic $g$ minimizes this distance locally near $g(t)$ if and only if

$$
g(t) \in M_{0} \text { or } g(t) \in P_{\mathrm{d}} \quad \text { and } \quad|\beta| \geqslant \pi .
$$

This fact is a direct consequence of the explicit expression of the distance on the cone $\mathcal{C}_{\alpha}$ that is given by

$$
\begin{aligned}
{\left[r_{1}^{2}+r_{2}^{2}-2 r_{1} r_{2} \cos \left(\left|x_{1}-x_{2}\right|\right)\right]^{1 / 2} } & \text { if }\left|x_{1}-x_{2}\right| \leqslant \pi, \\
r_{1}+r_{2} & \text { if }\left|x_{1}-x_{2}\right| \geqslant \pi,
\end{aligned}
$$

where $\left|x_{1}-x_{2}\right|$ is the distance in $\mathbb{R} / \alpha \mathbb{Z}$.

Using this expression, for any geodesic $g$ on the cone we have the following inequality:

$$
d(g(t), p) \geqslant\left|t-r_{0}\right| \quad \forall t .
$$

Since we aim to study the set of all the geodesics on an ESCS, it is helpful to first address the topological nature of this set. 


\subsubsection{Topology of the set of geodesics}

By definition, a geodesic of length $T$ is an element of $\mathcal{C}^{0}([0, T], M)$. The norm of uniform convergence gives us a topology on all the following sets.

\section{Definition 2.3.}

(i) For any subset $N$ of $M$, we denote by $\Gamma_{T}(N)$ the set of all the geodesics of length $T$ whose starting point is in $N$. The notation $\Gamma_{T}$ will be a shortcut for $\Gamma_{T}(M)$.

(ii) Given any (ordered) finite sequence of diffractive conical points $[p]=\left[p_{i_{1}}, \ldots, p_{i_{n}}\right]$, we denote by $\Gamma_{T}^{[p]}$ the set of all the geodesics of length $T$ having exactly $n$ diffractive conical points such that $p_{g, j}=p_{i_{j}}$. The set corresponding to non-diffractive geodesics will be denoted by $\Gamma_{T}^{0}$.

\section{Remarks.}

(i) These sets may be empty. For instance, if the sequence $[p]$ has at least two elements, the sets $\Gamma_{T}^{[p]}$ are empty for small $T$.

(ii) Consider a geodesic $g$ of $\Gamma_{T}^{[p]}$; any other geodesic $g^{\prime}$ in $\Gamma_{T}^{[p]}$ close to $g$ is uniquely determined by its starting point and its last diffraction angle. This parametrization shows that, for any sequence $[p]$, and any time $T$, the set $\Gamma_{T}^{[p]}$ is a three-dimensional manifold.

The following theorem and its corollary establish the topological nature of $\Gamma_{T}(N)$.

Theorem 2.4. For any $T, \Gamma_{T}(N)$ is compact if and only if $N$ is compact. In particular, if $M$ is compact, $\Gamma_{T}(M)$ is compact.

Corollary 2.5. The set $\Gamma_{T}(M)$ is always complete.

Proof. As in the smooth case, such a theorem is a direct consequence of Ascoli's theorem [3] and of the fact that a limit of geodesics is still a geodesic. The proof of this latter point consists here in two steps. We first address what happens away from the diffractive points (the proof then runs as in the smooth case). We then show that $g^{-1}(P)$ is still discrete when $g$ is a limit of geodesics. This is ensured by the inequality $(2.2)$.

We now want to study in more detail the set $\Gamma_{T}$ and in particular the stratification of it by the $\Gamma_{T}^{[p]}$. This is the goal of the following section. We will also completely forget about $\mathcal{C}^{0}$ : from now on every topological statement is to be understood in $\Gamma_{T}(M)$ (equipped with the topology of uniform convergence).

\section{Classical complexity}

As we already have pointed out, the geometry of one $\Gamma_{T}^{[p]}$ is rather simple, so the local geometry of $\Gamma_{T}$ only depends on the way these sets are close one to another. In order to understand this we introduce the following definition. 
Definition 3.1. The classical complexity of a geodesic $g$ is the number of sequences of diffractive points $[p]$ such that

$$
g \in \operatorname{Clos}\left(\Gamma_{T}^{[p]}\right)
$$

we denote it by $c_{\mathrm{c}}(g)$.

\section{Remarks.}

(i) The number $c_{\mathrm{c}}(g)$ is the answer to the following question.

How many sequences (possibly empty) of diffractive conical points $[p]$ are there such that there exists a sequence $\left(g_{n}\right)_{n \in \mathbb{N}} \in\left(\Gamma_{T}^{[p]}\right)^{\mathbb{N}}$ converging to $g$ ?

(ii) For fixed $T$ and $g$, the number of possible sequences $[p]$ is bounded, and thus we have the following equivalence:

$$
c_{\mathrm{c}}(g)=1 \Leftrightarrow g \in \operatorname{Int}\left(\Gamma_{T}^{[p]_{g}}\right)
$$

(we recall that the Int is taken relative to $\Gamma_{T}$ ).

(iii) A priori, this definition has nothing to do with the complexity of an orbit in a polygonal billiard [9, p. 63].

(iv) The case of non-diffractive geodesics is easily handled. For any $T$ we find that $\Gamma_{T}^{0}$ is open. Equivalently, if $g$ is a non-diffractive geodesic, then $c_{\mathrm{c}}(g)=1$.

Consider a sequence $g_{n}$ converging to $g$. There exist $\varepsilon$ and $n_{0}$ such that, for all $n \geqslant n_{0}$ and for all $i$, the restriction of $g_{n}$ to the interval $\left[t_{g, i}-\varepsilon, t_{g, i}+\varepsilon\right]$ can be identified with a geodesic on the corresponding cone. A consequence of the non-diffractive case is that, on the complement of these intervals, for large $n, g_{n}$ cannot have any diffraction. Thus the sequence $[p]$ such that $g \in \operatorname{Clos}\left(\Gamma_{T}^{[p]}\right)$ can only be obtained by deleting some of the diffractive points in $[p]_{g}$. Before addressing a general geodesic, it is instructive to study some examples in detail.

Example 3.2 (angles of $\pm \pi$ are special). In a plane wedge of angle $\alpha \neq \pi / k$, consider an incoming ray $\gamma$ hitting the tip of the sector, and consider the two families of parallel rays 'above' and 'under' $\gamma$. In each family, every ray will make the same reflections and eventually leave a neighbourhood of the vertex following the same direction. This gives two geodesics consisting of $\gamma$ followed by the outgoing ray parallel to one of these directions. We denote these geodesics by $g^{ \pm}$. Along these geodesics, the diffraction angle is $\pm \pi$, as can clearly be seen by unfolding the orbit (see Figure 2).

These two diffractive geodesics are, by definition, limits of non-diffractive ones. Conversely, consider a sequence of non-diffractive geodesic converging to $\gamma$ on some small interval $(a, b)$. This sequence can be decomposed into two subsequences depending on which side of the wedge the geodesic hits first. Since the sequence converges to $\gamma$ on $(a, b)$, one of these subsequences converges to $g^{+}$and the other to $g^{-}$. If the sequence is 

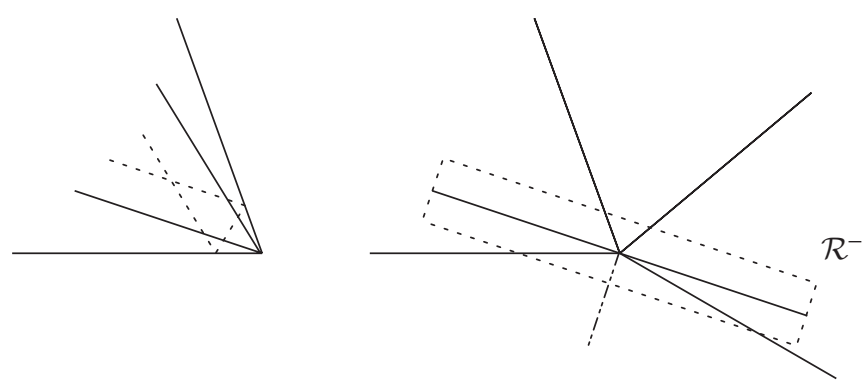

Figure 2. Definition of $g^{-}$.

known to converge, then only one of the two subsequences can be infinite and the limit is either $g^{+}$or $g^{-}$.

This example gives the complete classification on the cone by doubling the wedge.

Proposition 3.3. Let $g$ be a geodesic on the Euclidean cone of angle $\alpha(\neq 2 \pi / k)$. Then $c_{\mathrm{c}}(g)=2$ if and only if either $g$ begins (or ends) at $p$ or $g$ is diffractive in its interior with $\beta= \pm \pi$.

\section{Remarks.}

(i) The notion of diffraction angle is not well defined for an incoming (or outgoing) ray. However, such a geodesic is always a limit of non-diffractive geodesics; for instance, the outgoing ray defined by $g(t)=\left(t, x_{0}\right)$ is the limit of the family $\left(g_{\varepsilon}\right)_{\varepsilon>0}$ defined by $g_{\varepsilon}(t)=\left(t+\varepsilon, x_{0}\right)$.

(ii) Since the diffraction angle is $\pm \pi$, there is a continuous choice of a normal vector $\boldsymbol{n}(t)$ along $g^{+}$such that the mapping $j(t, s)=g^{+}(t)+\boldsymbol{s} \boldsymbol{n}(t)$ is well defined on $\mathbb{R}^{2} \backslash\left\{\left(t^{+}, s\right), s \geqslant 0\right\}$. Moreover, $j$ a local isometry into $\check{\mathcal{C}}_{\alpha}$.

(iii) This example shows that, on a general ESCS, diffraction angles of $\pm \pi$ will play a special role (for instance, see the following lemma).

Lemma 3.4. Let $g$ be a geodesic having all its diffraction angles different from $\pm \pi$. Then $c_{\mathrm{c}}(g)=1$.

Proof. We can find a $\varepsilon$ such that, on each interval $\left[t_{g, i}-\varepsilon, t_{g, i}+\varepsilon\right], g_{n}$ is a geodesic of the corresponding cone converging to a diffractive geodesic whose angle of diffraction is not $\pm \pi$. Necessarily, for large $n, g_{n}$ is thus diffractive at this conical point.

Example 3.5 (rectangles with slits). We want to generalize the first example by considering geodesics with several diffractions, such that each diffraction angle is $\pm \pi$. Let $g$ be such a geodesic. We first show that a rectangle with slits can be put around $g$. This is done by matching the local isometries $j$ constructed for each diffraction (see remark (ii) above). More precisely, if the sequence of diffraction angle is $\left(\varepsilon_{i} \pi\right)_{i}$ we let

$$
\mathcal{R}=[0, T] \times]-\delta, \delta\left[\backslash \cup S_{i},\right.
$$




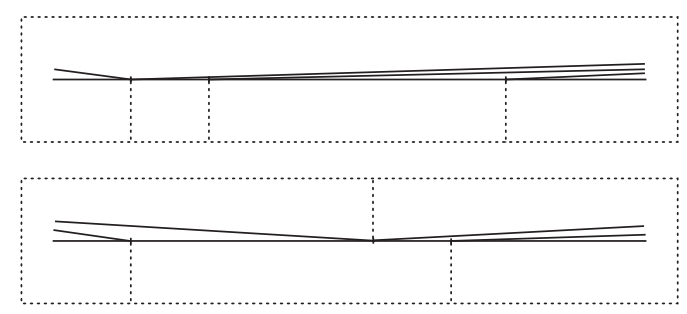

Figure 3. Rectangles with slits.

where $S_{i}$ is the segment (slit) $\left\{\left(t_{g, i}, s\right) \mid 0 \leqslant \varepsilon_{i} s<\delta\right\}$. There is a continuous choice of a normal vector $\boldsymbol{n}(t)$ along $g$ such that, for $\delta$ small enough, the mapping

$$
\begin{aligned}
j: \mathcal{R} & \rightarrow M \backslash P_{\mathrm{d}}, \\
(t, s) & \mapsto g(t)+s \boldsymbol{n}(t)
\end{aligned}
$$

is a local isometry. This construction is summarized in the following lemma.

Lemma 3.6. Let $g$ be a geodesic such that $\beta_{g, i}=\varepsilon_{i} \pi$ with $\varepsilon_{i}= \pm 1$. Then a neighbourhood of $g$ can be identified with the rectangle with slits $\mathcal{R}$ via the local isometry $j$ that is constructed above.

Remark 3.7. We have made the choice that a diffraction angle of $+\pi$ or $-\pi$ corresponds to an upward or downward slit, respectively. See Figure 3 for examples of such rectangles with slits.

Lemma 3.6 will allow us to compute simply the classical complexity of a geodesic such that all the diffraction angles are $\pm \pi$, since it says that we can do this computation in the rectangle $\mathcal{R}$. For instance, if the sequence of diffraction angles is $(\pi, \pi, \pi)$ we can construct approaching sequences of geodesics with the following diffractions: none, $\left[p_{1}\right],\left[p_{2}\right],\left[p_{3}\right],\left[p_{1}, p_{2}\right],\left[p_{2}, p_{3}\right],\left[p_{1}, p_{2}, p_{3}\right]$ and thus $c_{\mathrm{c}}(g)=7$. If the sequence of diffraction angles is $(-\pi, \pi,-\pi)$, the possible diffraction sequence for an approaching sequence of geodesics is $\left[p_{1}, p_{2}\right],\left[p_{1}, p_{2}, p_{3}\right],\left[p_{2}\right],\left[p_{2}, p_{3}\right]$ and thus $c_{\mathrm{c}}(g)=4$.

Using these rectangles with slits, we can also find the diffractive geodesics that are limits of non-diffractive ones.

Lemma 3.8. A geodesic $g$ is a limit of non-diffractive ones if and only if the sequence of its diffraction angles can be written

$$
(\underbrace{\varepsilon_{0} \pi, \ldots, \varepsilon_{0} \pi}_{k_{0}}, \underbrace{-\varepsilon_{0} \pi, \ldots,-\varepsilon_{0} \pi}_{k_{1}})
$$

with $\varepsilon_{0}= \pm 1$ and $k_{0}$ and $k_{1}$ possibly zero.

Proof. We already know that all the diffraction angles must be $\pm \pi$, so, using Lemma 3.6 we can identify a neighbourhood of $g$ with a rectangle with slits $\mathcal{R}$. In any other case than those given in the lemma, the position of the slits in the rectangle $\mathcal{R}$ 
forbids the existence of a sequence of non-diffractive geodesics approaching $g$. Conversely, if the sequence of diffraction angles is as in the lemma, such a sequence is easily constructed.

These examples show that not only the angles of $\pm \pi$ but also the place they occupy in the sequence of diffraction angles plays a special role in computing the classical complexity. Among all geodesics, those having all their diffraction angles equal to $\pi$ (or $-\pi$ ) are special. They are studied in the following section.

\subsection{Geodesics $g^{ \pm}$}

We begin by constructing geodesics having only $\pi($ or $-\pi)$ as diffraction angles. We start from a point $m$ in direction $v$; the geodesic is non-diffractive for small times. If it reaches a conical point, we define $t_{1}^{+}=t_{1}^{-}$as the first time this happens. We continue the geodesic in two ways, making angle $\pm \pi$. This gives two geodesics: $g^{ \pm}$. Each of these is defined until it reaches another conical point. If $g^{+}$reaches a second diffractive point, we denote by $t_{2}^{+}$the time it happens and continue $g^{+}$, making the angle of diffraction $+\pi$, and so on. We do the same with $g^{-}$. This construction gives, for any initial data $(m, v)$, two infinite geodesics $g^{ \pm}$with sequences of diffraction times $\left(t_{i}^{ \pm}\right)$and angles of diffraction $\beta_{g^{ \pm}, i}= \pm \pi$.

The existence of rectangles with slits along the geodesics $g^{ \pm}$allows the following proposition.

Proposition 3.9. For all starting points $m$ and all times $T$, there is only a finite number of directions $v_{i}$ such that the geodesic emanating from $m$ in the direction $v_{i}$ hits a diffractive point before the time $T$.

Proof. Take such a direction $v_{i}$. This gives rise to two geodesics, $g^{ \pm}$. For any time $T$, we construct two rectangles $\mathcal{R}^{ \pm}$along $g^{ \pm}(] 0, T[$ ) (see Example 3.5). Take another geodesic emanating from $m$; for small times it can be lifted to a small segment in $\mathcal{R}^{+}$and in $\mathcal{R}^{-}$. If the direction is close enough to $v_{i}$, this small segment can be extended to length $T$ without leaving the rectangles $\mathcal{R}^{ \pm}$. In one of these rectangles, it does not cross the slits, so the segment projects onto a non-diffractive geodesic. Since the set of directions is compact, there are only a finite number of directions that are diffractive before time $T$.

Proposition 3.9 implies the following two technical lemmas that will reduce the computation of the classical complexity to a combinatorial problem. The first lemma will show that if a sequence of $\Gamma_{T}^{[p]}$ converges to $g$ then $[p]$ is obtained from $[p]_{g}$ by deleting the first $k_{0}$ and the last $k_{1}$ conical points of $[p]_{g}$. The second lemma will then prove that the conical points deleted at the beginning must all have the same diffraction angle, which, moreover, is $\pm \pi$. An analogous statement is true for the conical points that are deleted at the end (see Figure 4).

Lemma 3.10. Let $\left(g_{n}\right)$ be a sequence of geodesics of length $T$ converging to $g$ and such that, for some $j_{0} \leqslant j_{1}$, there exist two sequences $\left(t_{n}^{0}\right)$ and $\left(t_{n}^{1}\right)$ converging to $t_{g, j_{0}}$ 
and $t_{g, j_{1}}$, respectively, such that

$$
\forall n, \quad g_{n}\left(t_{n}^{i}\right)=p_{g, j_{i}}, \quad i=0,1 .
$$

Then the following holds:

$$
\exists n_{0}, \quad \forall n>n_{0}, \quad g_{n}(t)=g\left(t-t_{n}^{0}+t_{g, j_{0}}\right) \text { on }\left[t_{n}^{0}, t_{n}^{1}\right] .
$$

In particular, for $n>n_{0}, g_{n}$ is also diffractive at $p_{g, j}$ for every $j_{0} \leqslant j \leqslant j_{1}$.

Proof. On $\left[t_{n}^{0}, T\right], g_{n}$ is a geodesic emanating from $p_{g, j_{0}}$ that is diffractive at some time $t_{n}^{1}$. Since $g_{n}$ converges to $g$, Proposition 3.9 shows that, for large $n, g_{n}$ and $g$ follow the same outgoing ray at $p_{g, j_{0}}$. If $j_{1}=j_{0}+1$, the conclusion then holds; otherwise, we can iterate the argument starting from $p_{g, j_{0}+1}$.

Lemma 3.11. Let $g$ be a geodesic emanating from $m$ in the direction $v$ that reaches a diffractive point before time $T$. Let $\left(g_{n}\right)$ be a sequence of geodesics of length $T$ emanating from $m$, non-diffractive on $] 0, T]$ and such that

$$
\exists 0<a<b \mid g_{n \mid[a, b]} \rightarrow g_{\mid[a, b]} .
$$

Then the geodesics $g^{ \pm}$emanating from $(m, v)$ are the only accumulation points of the sequence $\left(g_{n}\right)$.

Proof of Lemma 3.11. We put rectangles $\mathcal{R}^{ \pm}$along $g^{ \pm}$, respectively. For $n$ large enough, each $g_{n}$ corresponds to a segment in each rectangle but only in one does it not cross the slits. Since we are dealing with segments, convergence on $[a, b]$ implies convergence on $[0, T]$ and we are done.

\section{Remarks.}

(i) A symmetrical statement is true for geodesics ending in $m$.

(ii) The assumption on the length of $g_{n}$ can be relaxed if we know that $g_{n}$ does not coincide with $g$. Indeed, using the rectangles $\mathcal{R}^{ \pm}$once again, it can be shown that any geodesic that does not coincide with $g_{\mid[a, b]}$, but that is sufficiently close to $g_{\mid[a, b]}$, can be uniquely extended to a non-diffractive geodesic defined on $[a, T]$.

(iii) We remind the reader that a limit of non-diffractive geodesics is not necessarily a geodesic of type $g^{ \pm}$(see Lemma 3.8). The assumption that all the geodesics $g_{n}$ emanate from $m$ deals with this point.

These two lemmas lead to the computation of the classical complexity. 


\subsection{Classical complexity: computation}

Consider a geodesic $g$ of length $T$ with $n$ diffractive points and assume that $g$ is in $\operatorname{Clos}\left(\Gamma_{T}^{[p]}\right)$, the first diffractive point in $[p]$ is some $p_{g, j_{0}}$, and the last one is some $p_{g, j_{1}}$ with $j_{1} \geqslant j_{0}$. Lemma 3.10 implies that, since there exists a sequence of $\Gamma_{T}^{[p]}$ converging to $g$ then, necessarily, $[p]=\left[p_{g, j_{0}}, p_{g, j_{0}+1} \cdots p_{g, j_{1}}\right]$. Then, using Lemma 3.11, we show that

$$
\exists \varepsilon_{0}, \varepsilon_{1} \in\{+,-\} \mid \forall j<j_{0}, \beta_{g, j}=\varepsilon_{0} \pi \text { and } \forall j>j_{1}, \beta_{g, j}=\varepsilon_{1} \pi .
$$

Conversely, suppose that $j_{0}$ and $j_{1}$ exist such that $j_{0} \leqslant j_{1}$ and satisfy condition (3.2). Then we can claim that there exists a sequence of geodesics in $\Gamma_{T}^{\left[p_{g, j_{0}}, \ldots, p_{g, j_{1}}\right]}$ converging to $g$. Indeed, on $\left[t_{g, j_{1}}, T\right],(3.2)$ implies that $g$ is of type $\varepsilon_{1}$ and is thus a limit of nondiffractive rays emanating from $p_{g, j_{1}}$. The same is true on $\left[0, t_{g, j_{0}}\right]$ since, on this interval, $g$ is of type $\varepsilon_{0}$. The concatenation of a ray coming into $p_{g, j_{0}}$ followed by $g$ until $p_{g, j_{1}}$ followed by a ray emanating from $p_{g, j_{1}}$ forms a geodesic that can be as close to $g$ as is desired. This construction can clearly be seen using a rectangle with slits along the beginning and along the end of the geodesic (see Figure 4).

Finally, the families of approaching geodesics are obtained by avoiding the first $j_{0}-1$ and the last $n-j_{1}$ diffractive points (see also Figure 4). Computing the classical complexity thus amounts to enumerating the couples $\left(j_{0}, j_{1}\right)$ satisfying $(3.2)$ and addressing the possibility for $g$ to be a limit of non-diffractive geodesics (which has been done in Lemma 3.8).

It is always possible to write the sequence of diffraction angles in the following way:

$$
(\underbrace{\varepsilon_{0} \pi, \ldots, \varepsilon_{0} \pi}_{k_{0}}, \beta_{g, l_{0}}, \ldots, \beta_{g, l_{1}}, \underbrace{\varepsilon_{1} \pi, \ldots, \varepsilon_{1} \pi}_{k_{1}}), \quad \beta_{g, l_{i}} \neq \varepsilon_{i} \pi
$$

where the subsequence $\beta_{g, l_{0}}, \ldots, \beta_{g, l_{1}}$ may be empty. This latter case corresponds to the geodesics that are limits of non-diffractive geodesics and we say that $g$ is of empty type.

We then have the following discussion.

(1) The geodesic $g$ is not of empty type. Condition (3.2) is then equivalent to $j_{0} \leqslant l_{0}$ and $j_{1} \geqslant l_{1}$. Since the geodesic is not a limit of non-diffractive geodesics, we have

$$
c_{\mathrm{c}}(g)=\left(k_{0}+1\right)\left(k_{1}+1\right) \text {. }
$$

(2) The geodesic is of empty type and $k_{0} k_{1} \neq 0$. The geodesic is a limit of non-diffractive ones and (3.2) is equivalent to

$$
j_{0} \leqslant k_{0}+1, \quad j_{1} \geqslant k_{1}+1, \quad j_{1} \geqslant j_{0} .
$$

There are $\left(k_{0}+1\right)\left(k_{1}+1\right)-1$ pairs satisfying this condition. Adding the non-diffractive geodesics, we find

$$
c_{\mathrm{c}}(g)=\left(k_{0}+1\right)\left(k_{1}+1\right) .
$$

(3) The geodesic is of empty type and $k_{0} k_{1}=0$. The geodesic is then a limit of nondiffractive geodesics and (3.2) is equivalent to $j_{0} \geqslant j_{1}$. This gives $\frac{1}{2} n(n+1)$ pairs and the following complexity:

$$
c_{\mathrm{c}}(g)=\frac{1}{2} n(n+1)+1 .
$$




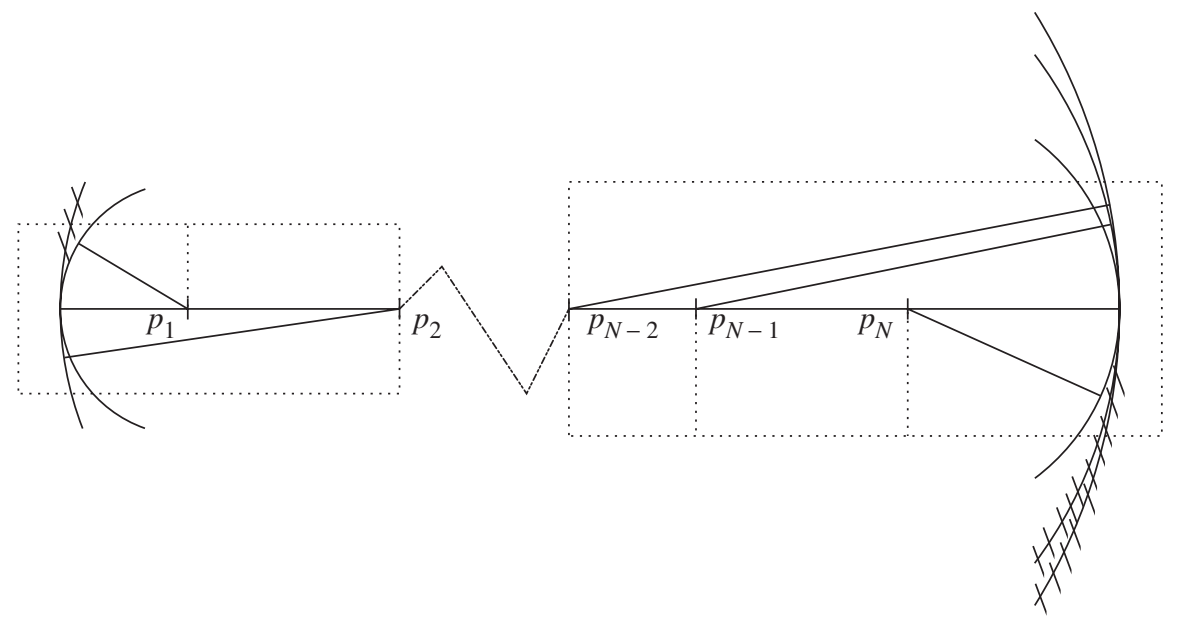

Figure 4. An example.

We resume these computations in the following theorem.

Theorem 3.12. Let $g$ be a geodesic of length $T$ with $n$ diffractive points and such that its sequence of diffraction angles is written in the form (3.3). One of the following then happens:

(i) $g$ is not of empty type and $c_{\mathrm{c}}(g)=\left(k_{0}+1\right)\left(k_{1}+1\right)$;

(ii) $g$ is of empty type and $k_{0} k_{1} \neq 0$ so then $c_{\mathrm{c}}(g)=\left(k_{0}+1\right)\left(k_{1}+1\right)$;

(iii) $g$ is of empty type and $k_{0} k_{1}=0$ so then $c_{\mathrm{c}}(g)=\frac{1}{2} n(n+1)+1$.

In Figure 4 we have represented the rectangles with slits along the beginning and along the end of the geodesic. The approaching geodesics are obtained by following some ray missing the first diffractive points, then the geodesic itself and then some ray emanating from another diffractive point that misses the last diffractive points. The circles correspond to the locus of the starting points and endpoints of the geodesics belonging to one particular family of approaching geodesics (the hatched part corresponds to points of the circle that cannot be attained because of the slits). They also correspond to the projection of the Lagrangian submanifolds described in $\S 4.2$, below.

\section{Applications}

We will give two straightforward applications of the previous discussion. The first one describes the periodic (eventually diffractive) geodesics of an ESCS, and the second one describes geometrically what is to be understood as the canonical relation associated with the (diffractive) geodesic flow on an ESCS. 


\subsection{Periodic geodesics}

One question of interest (in particular when aiming to prove some kind of trace formula [4]) is to know whether a given periodic geodesic is isolated or part of a family. We state the proposition in the case when $M$ is oriented (see the remark (i) after it for the non-oriented case).

Proposition 4.1. Let $g$ be a periodic geodesic of length $T$ of an oriented ESCS. One of the following then occurs.

(i) The geodesic $g$ is non-diffractive; it is then interior to a family of non-diffractive periodic geodesics of the same length.

(ii) All the angles of diffraction are $\pi$ (or $-\pi$ ); $g$ is then the boundary of a family described in the first case.

(iii) In any other case, $g$ is isolated in the set of periodic geodesics.

Proof. In the first case everything happens in $M_{0}$, where the metric is Euclidean. Since $M$ is oriented, the normal vector to $g$ is well defined and the geodesics $g_{\varepsilon}(t)=g(t)+\varepsilon \boldsymbol{n}(t)$ are periodic and of the same length. In the second case we use a rectangle of type \pm , and we can define geodesics parallel to $g$ by using the same argument as in the non-diffractive case. The only difference is that, because of the slits, $\varepsilon$ must be of chosen sign. In the third case, assume first that $g$ has a diffraction angle $\beta_{g, j}$ different from $\pm \pi$. Consider an approaching sequence $\left(g_{n}\right)$ of periodic geodesics. All these geodesics must go through $p_{g, j}$ at times $t_{n}+k T$. Lemma 3.10 implies that, for large $n, g_{n}$ and $g$ coincide. If all the angles of $g$ are $\pm \pi$ but not all of the same sign (which is addressed by case (ii)), then $g$ (or its double) is not of empty type. Consequently, there is also one conical point through which any approaching sequence of geodesics must go. Repeating the preceding argument gives the conclusion.

\section{Remarks.}

(i) If the surface is not orientable, then in the first two cases a neighbourhood of geodesic can cease to be orientable. The argument then breaks down and $g$ is isolated. In this case, the former proposition remains true for the double of $g$.

(ii) The existence of a rectangle $\mathcal{R}^{ \pm}$along a geodesic $g$ of the second type implies that the only periodic geodesics of period bounded by some $T^{\prime}$ close to $g$ are the non-diffractive geodesics of the corresponding family. This isn't true anymore if you allow the period to go to infinity. In fact, there are translation surfaces for which the geodesics emanating from a given point are periodic for a dense set of directions, in which case one can find a sequence $g_{n}$ of periodic geodesics (of period $\left.T_{n} \rightarrow \infty\right)$ such that

$$
\forall T, \forall \varepsilon \exists n_{0} \mid \sup _{[0, T]} d\left(g_{n}(t), g(t)\right) \leqslant \varepsilon .
$$


(iii) We could define a notion of classical complexity for a periodic geodesic by asking the following question. For a given periodic geodesic $g$ of period less than $T$, how many different types of periodic geodesics of period less than $T$ can approach $g$ ? The proposition answers this question, but it tells more since it also proves that 'most' diffractive periodic geodesics will be isolated.

One interesting question is as follows. Given an ESCS, how complex can the classical complexity be? The following proposition answers this question (at least partly).

Proposition 4.2. Let $M$ be an ESCS such that there exists a non-diffractive periodic orbit. Then, for any given $N$, there is a geodesic $g$ such that $c_{\mathrm{c}}(g)=N$.

Proof. The existence of a non-diffractive periodic orbit implies the existence, at the boundary of the corresponding family, of a periodic diffractive geodesic $g$ such that all its diffraction angles are $\pi$. We will construct a geodesic having a sequence of diffractions angles written in the form (3.3) with arbitrary $k_{0}$ and $k_{1}$. We pick a point on $g$, and begin by following $g$ for a time long enough to have $k_{0}$ diffraction angles. We then leave $g$ and go to another diffractive point; we follow then some diffractive geodesic that comes back to $g$ and follow again $g$ enough time to have $k_{1}$ diffractions. This gives the desired geodesic.

The next application is concerned with symplectic aspects associated with $\Gamma_{T}$.

\subsection{Symplectic aspects}

The set $\Gamma_{T}$ gives a relation $\Lambda_{T}$ from $T^{*}\left(M_{0}\right)$ to itself which is defined by

$$
\begin{aligned}
\Lambda_{T}=\left\{\left(m_{1}, m_{0}, \mu_{1}, \mu_{0}\right) \in T^{*}\left(M_{0}\right) \times T^{*}\left(M_{0}\right)\left|\exists g \in \Gamma_{T},\right| \mu_{0} \mid\right. & =\left|\mu_{1}\right|, \\
g(0)=m_{0}, \mu_{0}=\left|\mu_{0}\right|\left\langle g^{\prime}(0), \cdot\right\rangle_{m_{0}}, g(T)=m_{1}, \mu_{1} & \left.=\left|\mu_{1}\right|\left\langle g^{\prime}(T), \cdot\right\rangle_{m_{1}}\right\},
\end{aligned}
$$

where $\langle\cdot, \cdot\rangle_{m}$ is the Euclidean scalar product in $T_{m} M_{0}$ and $|\cdot|$ is the associated norm.

Remark 4.3. The same definition on a smooth Riemannian manifold makes $\Lambda_{T}$ the canonical relation associated with the geodesic flow at time $T$.

Given any subset $\mathcal{V}$ of $\Gamma_{T}$ we can define $\Lambda_{T}^{\mathcal{V}}$ by specifying, in the definition of $\Lambda_{T}$, that the geodesic $g$ belongs to $\mathcal{V}$. The classical complexity and the constructions made in the previous sections allow us to prove the following proposition.

Proposition 4.4. Given any geodesic of length $T$ starting and ending in $M_{0}$, there exist $c_{\mathrm{c}}(g)$ Lagrangian submanifolds $\Lambda_{g, i}$ of $T^{*}\left(M_{0}\right) \times T^{*}\left(M_{0}\right)$ such that, for any sufficiently small neighbourhood $\mathcal{V}$ of $g$, we have the following inclusion:

$$
\Lambda_{T}^{\mathcal{V}} \subset \bigcup_{1}^{c_{c}(g)} \Lambda_{g, i}
$$

Furthermore, each $\Lambda_{g, i}$ is determined by an explicit phase function and there exists $\Sigma$ such that, for $i \neq j$,

$$
\Lambda_{g, i} \cap \Lambda_{g, j}=\Sigma,
$$

the intersection being clean. 
Proof. We take a geodesic $g$ and $[p]$ such that $g \in \operatorname{Clos}\left(\Gamma_{T}^{[p]}\right)$ (by definition of $c_{\mathrm{c}}(g)$ there are $c_{\mathrm{c}}(g)$ choices for $\left.[p]\right)$. We will construct a Lagrangian submanifold associated with $[p]$. We begin by assuming $[p] \neq \emptyset$; there exist $j_{0}$ and $j_{1}$ such that

$$
[p]=\left[p_{g, j_{0}}, \ldots p_{g, j_{1}}\right]
$$

and there exist $\varepsilon_{0}$ and $\varepsilon_{1}$ such that

$$
\begin{aligned}
& \forall j<j_{0}, \quad \beta_{g, j}=\varepsilon_{0} \pi, \\
& \forall j>j_{1}, \quad \beta_{g, j}=\varepsilon_{1} \pi .
\end{aligned}
$$

Each geodesic in $\Gamma_{T}^{[p]}$ consists of a ray coming in $p_{g, j_{0}}$, the portion of $g$ between $p_{g, j_{0}}$ and $p_{g, j_{1}}$, and a ray coming out $p_{g, j_{1}}$. There is a rectangle of type $\left(\varepsilon_{0}\right)$ around the first diffractive points (if $p_{g, j_{0}}$ is the first diffractive point, then the rectangle has no slits) and a local isometry $j^{\varepsilon_{0}}$ such that $j^{\varepsilon_{0}}\left(t_{g, j_{0}}, 0\right)=p_{g, j_{0}}$. We can define $d_{0}\left(\cdot, p_{g, j_{0}}\right)$ in a small neighbourhood of $m_{0}$ by

$$
d_{0}\left(m, p_{g, j_{0}}\right)=d_{\mathbb{R}^{2}}\left(\left(j^{\varepsilon_{0}}\right)^{-1}(m),\left(t_{g, j_{0}}, 0\right)\right) .
$$

The same construction around the end of $g$ gives $d_{1}\left(p_{g, j_{1}}, \cdot\right)$ defined in a neighbourhood of $m_{1}$. The phase function

$$
\left[d_{0}\left(m, p_{g, j_{0}}\right)+t_{g, j_{1}}-t_{g, j_{0}}+d_{1}\left(p_{g, j_{1}}, m^{\prime}\right)-T\right] \theta
$$

defines a Lagrangian submanifold that includes the part of $\Lambda_{T}$ corresponding to the geodesics of $\Gamma_{T}^{[p]}$ close to $g$. For the non-diffractive geodesics close to $g$ one should take as a phase function

$$
\left[d_{\mathbb{R}^{2}}\left(j^{-1}(m), j^{-1}\left(m^{\prime}\right)\right)-T\right] \theta,
$$

where $j$ is the local isometry between the rectangle of type

$$
(+), \quad(-) \text { or }(\varepsilon, \ldots, \varepsilon,-\varepsilon, \ldots,-\varepsilon)
$$

and a neighbourhood of $g$. In the definition of these Lagrangian submanifolds, we have not taken the slits into account, so in fact the geodesics corresponding to $\Gamma_{T}$ form a subset of the corresponding Lagrangian submanifold. The set $\Sigma$ corresponds to the geodesic $g$. We described using the following coordinates of $T^{*} M_{0}:\left(x_{0}, y_{0}, \xi_{0}, \eta_{0}\right)$ near the beginning of the geodesic $g$ and $\left(x_{1}, y_{1}, \xi_{1}, \eta_{1}\right)$ such that $g$ corresponds to $y_{i}=0$. The intersection $\Sigma$ is then parametrized by

$$
\Sigma=\left\{\left(x_{1}, y_{1}=0, \xi_{1}, \eta_{1}=0, x_{0}, y_{0}=0, \xi_{0}, \eta_{0}=0\right) \mid x_{1}+x_{0}=T, \xi_{1}=\xi_{0}\right\} .
$$

The fact that the intersections are clean is straightforward once good coordinates are chosen.

In Figure 4, the conormal sets to the circles correspond to some of the Lagrangian submanifolds of Proposition 4.4. 
This proposition is very important, since it describes what should be taken as the generalization of the geodesic flow (as long as propagation of singularities for the wave equation is considered). It also gives the geometric wavefront. In particular we would like to know whether the propagator for the wave equation is a Fourier integral operator and with which canonical transformation it is associated. This study implies that if $c_{\mathrm{c}}(g)>1$, then the propagator is not in this class of operators.

\section{References}

1. J. Cheeger and M. TAYlor, On the diffraction of waves by conical singularities, I, Commun. Pure Appl. Math. 35 (1982), 275-331.

2. J. CheEger and M. TAYlor, On the diffraction of waves by conical singularities, II, Commun. Pure Appl. Math. 35 (1982), 487-529.

3. J. Dixmier, Topologie générale (Presses Universitaires de France, Paris, 1981).

4. J. J. Duistermant and V. W. Gulllemin, The spectrum of positive elliptic operators and periodic bicharacteristics, Invent. Math. 29 (1975), 39-79.

5. F. G. Friedlander, On the wave equation in plane regions with polygonal boundary, in Advances in microlocal analysis (Lucca, 1985), NATO Advanced Science Institutes Series, pp. 135-150 (Springer, 1986).

6. V. Guillemin and R. Melrose, The Poisson summation formula for manifolds with boundary, Adv. Math. 32 (1979), 204-232.

7. P. Hubert And T. A. Schmidt, Invariants of translation surfaces, Annls Inst. Fourier 51 (2001), 461-495.

8. A. KAтOK, The growth rate for the number of singular and periodic orbits for a polygonal billiard, Commun. Math. Phys. 111 (1987), 151-160.

9. S. Tabachnikov, Billiards, Panoram. Synthèses 1 (1995).

10. M. Troyanov, Les surfaces euclidiennes à singularités coniques, Enseign. Math. 32 (1986), 79-94.

11. J. Wunsch, A Poisson relation for conic manifolds, Math. Res. Lett. 9 (2002), 813-828. 